TITLE:

\title{
Potential distribution of a nonuniformly charged ellipsoid
}

AUTHOR(S):

Kiwamoto, Y; Aoki, J; Soga, Y

CITATION:

Kiwamoto, Y ...[et al]. Potential distribution of a nonuniformly charged ellipsoid. PHYSICS OF PLASMAS 2004, 11(10): 4868-4870

ISSUE DATE:

2004-10

URL:

http://hdl.handle.net/2433/50231

\section{RIGHT:}

Copyright 2004 American Institute of Physics. This article may be downloaded for personal use only. Any other use requires prior permission of the author and the American Institute of Physics. 


\title{
Potential distribution of a nonuniformly charged ellipsoid
}

\author{
Y. Kiwamoto, ${ }^{\text {a) }}$ J. Aoki, and Y. Soga \\ Graduate School of Human and Environmental Studies, Kyoto University, Yoshida Nihonmatsu-cho, \\ Sakyo-ku, Kyoto 606-8501, Japan
}

(Received 20 May 2004; accepted 24 June 2004; published online 28 September 2004)

\begin{abstract}
A convenient formula is obtained for fast calculation of the three-dimensional potential distribution associated with a spatially varying charge-density distribution by reconstructing it as a superposed set of nested spheroidal shells. It is useful for experimental analyses of near-equilibrium states of non-neutral plasmas and for quick evaluation of the gravity field associated with stellar mass distributions. (C) 2004 American Institute of Physics. [DOI: 10.1063/1.1783878]
\end{abstract}

In experimental analyses of dynamics and equilibria of non-neutral plasmas it is always necessary to know the potential distribution associated with an observation-based charge-density distribution. ${ }^{1}$ A scheme of fast numerical procedure is available for two-dimensional configurations. ${ }^{2}$ For three-dimensional (3D) cases, in general, we have to resort to direct integration of Poisson equation, which requires an enormous amount of time. ${ }^{3}$ Analytical expressions of the potential are known only for uniformly charged ellipsoids. ${ }^{4,5}$ They were obtained by employing a procedure originally developed for study of the gravity field. ${ }^{6}$ A full expression of the potential has been given explicitly by Mohri et al. for an elongated spheroid.

The purpose of this report is to obtain an analytical expression for calculating the 3D potential distribution associated with a realistic charge distribution. In analyzing experimental data of non-neutral plasmas, we have found that a wide class of 3D particle-density distributions can be described in terms of nested spheroidal shells or by superposing two or more sets of the nested shells. This observation has led us to initiate a task to derive an expression for the potential distribution associated with a charged spheroidal shell. This expression can be used as a Green's function for constructing the 3D potential from nested spheroids that constitute nonuniform charge distributions. It is useful also for quick calculation of the gravity potential associated with various shapes of mass distribution with axial symmetry.

We derive the Green's function for the shell by using the expressions for uniformly charged spheroids. We set the center of a spheroid at the origin of the cylindrical coordinate system with a rotational symmetry around the $z$ axis. For convenience of reference, we summarize expressions for the potential distribution $\phi^{s}(r, z)$ associated with a uniformly charged ellipsoid with the radius $a$ and the axial length $2 b$, therefore with the aspect ratio of $\alpha=b / a$. Particles charged with $q$ fill the ellipsoid with a uniform number density of $n$. Inside the spheroid the potential is given as ${ }^{4,5}$

$$
\phi^{s}(r, z ; a)=-\frac{q n}{4 \epsilon_{0}}\left\{[1-G(\alpha)] r^{2}+2 G(\alpha) z^{2}\right\} .
$$

Here the expression for $G(\alpha)$ is

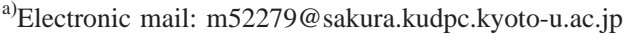

$$
\begin{aligned}
G(\alpha)= & -\frac{1}{\alpha^{2}-1} \\
& +\frac{\alpha}{2\left(\alpha^{2}-1\right)^{3 / 2}} \ln \left|\frac{\alpha+\left(\alpha^{2}-1\right)^{1 / 2}}{\alpha-\left(\alpha^{2}-1\right)^{1 / 2}}\right|(\alpha>1) \\
= & \frac{1}{1-\alpha^{2}}-\frac{\alpha}{\left(1-\alpha^{2}\right)^{3 / 2}} \tan ^{-1}\left(\frac{1}{\alpha^{2}}-1\right)^{1 / 2}(\alpha<1) .
\end{aligned}
$$

Outside the spheroid the potential is given as

$$
\begin{aligned}
\phi^{s}(r, z ; a) & \\
= & -\frac{q n a^{2}}{4 \epsilon_{0}} \alpha\left\{\frac{1}{\left(\alpha^{2}-1\right)^{1 / 2}}\right. \\
& \times \ln \left|\frac{\left(\alpha^{2}+\eta\right)^{1 / 2}-\left(\alpha^{2}-1\right)^{1 / 2}}{\left(\alpha^{2}+\eta\right)^{1 / 2}+\left(\alpha^{2}-1\right)^{1 / 2}} \frac{\alpha+\left(\alpha^{2}-1\right)^{1 / 2}}{\alpha-\left(\alpha^{2}-1\right)^{1 / 2}}\right| \\
& +\frac{x^{2}-2 y^{2}}{2\left(\alpha^{2}-1\right)^{3 / 2}} \ln \left|\frac{\left(\alpha^{2}+\eta\right)^{1 / 2}-\left(\alpha^{2}-1\right)^{1 / 2}}{\left(\alpha^{2}+\eta\right)^{1 / 2}+\left(\alpha^{2}-1\right)^{1 / 2}}\right| \\
& \left.+\frac{\left(\alpha^{2}+\eta\right) x^{2}-2(1+\eta) y^{2}}{\left(\alpha^{2}-1\right)(1+\eta)\left(\alpha^{2}+\eta\right)^{1 / 2}}\right\}
\end{aligned}
$$

for $\alpha>1,{ }^{7}$ and

$$
\begin{aligned}
\phi^{s}(r, z ; a)= & -\frac{q n a^{2}}{4 \epsilon_{0}} \alpha\left\{\frac { 2 } { ( 1 - \alpha ^ { 2 } ) ^ { 1 / 2 } } \left[\tan ^{-1}\left(\frac{\alpha^{2}+\eta}{1-\alpha^{2}}\right)^{1 / 2}\right.\right. \\
& \left.-\tan ^{-1}\left(\frac{\alpha^{2}}{1-\alpha^{2}}\right)^{1 / 2}\right]+\frac{x^{2}-2 y^{2}}{\left(1-\alpha^{2}\right)^{3 / 2}}\left[\frac{\pi}{2}\right. \\
& \left.-\tan ^{-1}\left(\frac{\alpha^{2}+\eta}{1-\alpha^{2}}\right)^{1 / 2}\right] \\
& \left.-\frac{\left(\alpha^{2}+\eta\right) x^{2}-2(1+\eta) y^{2}}{\left(1-\alpha^{2}\right)(1+\eta)\left(\alpha^{2}+\eta\right)^{1 / 2}}\right\}
\end{aligned}
$$

for $\alpha<1$. Here we have introduced normalized coordinates,

$$
x=r / a, y=z / a, \eta=\xi / a^{2} .
$$

The parameter $\xi$ satisfies the equation, $r^{2} /\left(a^{2}+\xi\right)+z^{2} /\left(b^{2}\right.$ $+\xi)=1$, so that it represents the distance from the spheroidal surface, and the explicit expression of $\eta$ is 


$$
\begin{aligned}
\eta= & \frac{1}{2}\left\{x^{2}+y^{2}-\alpha^{2}-1+\left[\left(x^{2}+y^{2}-\alpha^{2}-1\right)^{2}\right.\right. \\
& \left.\left.-4 \alpha^{2}\left(1-x^{2}-\frac{y^{2}}{\alpha^{2}}\right)\right]^{1 / 2}\right\} .
\end{aligned}
$$

To the authors' knowledge the expression for the potential outside an oblate spheroid is the first to be published.

Let us consider the potential generated by a uniformly charged spheroidal shell that extends from $r=a-\Delta a$ to $r=a$ in the $z=0$ plane with the aspect ratio of $\alpha$. If the point of interest lies inside the shell, the shell contribution is equal to the summation of $\phi^{s}(r, z ; a)$ and $\phi^{s}(r, z ; a-\Delta a)$ with the opposite sign of charge $q$, both evaluated with Eq. (1). It is readily known that the superposed potentials cancel out to zero. Therefore we only need to consider the contribution of charged shells that do not include the point $(r, z)$. Such contribution is given by Eq. (3) for shells with $\alpha>1$ or by Eq. (4) for shells with $\alpha<1$.

The contribution to the potential $\Delta \phi$ at $(r, z)$ from a spheroidal shell with the aspect ratio $\alpha$, radius $r=a$, and thickness $\Delta a$ on the $z=0$ plane may be written as

$$
\begin{aligned}
\Delta \phi(r, z ; a) & =\phi^{s}(r, z ; a)-\phi^{s}(r, z ; a-\Delta a) \\
& =\Delta a\left\{\frac{\partial \phi^{s}}{\partial a}+\frac{\partial \phi^{s}}{\partial \eta} \frac{\partial \eta}{\partial a}\right\} \\
& =q n g(r, z ; a, \alpha) \Delta a .
\end{aligned}
$$

Here we note that $\eta$ includes the radius $a$ as a normalizing factor in $x=r / a$ and $y=z / a$. Then the potential $\phi(r, z)$ at $(r, z)$ may be determined as

$$
\phi(r, z)=\int_{0}^{\rho_{c}} d \rho q n(\rho) g(r, z ; \rho, \alpha) .
$$

Here $q n(\rho)$ stands for the charge density on the shell characterized by the radius $\rho$ and the aspect ratio $\alpha(\rho)$. The upper bound $\rho_{c}$ stands for the radius of the outermost shell that excludes the point $(r, z)$.

The Green's function $g(r, z ; \rho, \alpha)$ is determined by introducing the external solution (3) and (4) into the equation,

$$
g(r, z ; \rho, \alpha)=\frac{1}{q n}\left\{\frac{\partial \phi^{s}}{\partial a}+\frac{\partial \phi^{s}}{\partial \eta} \frac{\partial \eta}{\partial a}\right\}_{a=\rho}
$$

Lengthy but straightforward calculations lead to the following expression:

$$
\begin{aligned}
g(r, z ; \rho, \alpha) & \\
= & -\frac{\alpha}{2 \epsilon_{0}}\left\{\frac{\rho}{\left(\alpha^{2}-1\right)^{1 / 2}}\right. \\
& \times \ln \left|\frac{\left(\eta+\alpha^{2}\right)^{1 / 2}-\left(\alpha^{2}-1\right)^{1 / 2}}{\left(\eta+\alpha^{2}\right)^{1 / 2}+\left(\alpha^{2}-1\right)^{1 / 2}} \frac{\alpha+\left(\alpha^{2}-1\right)^{1 / 2}}{\alpha-\left(\alpha^{2}-1\right)^{1 / 2}}\right| \\
& +\frac{1}{\left(\eta+\alpha^{2}\right)^{1 / 2}(\eta+1) \rho}\left(\rho^{2}-\frac{r^{2}}{\eta+1}-\frac{z^{2}}{\eta+\alpha^{2}}\right) \\
& \left.\times \frac{\left(\eta+\alpha^{2}+1\right) r^{2}+(\eta+1) z^{2}}{r^{2}+z^{2}-\left(2 \eta+\alpha^{2}+1\right) \rho^{2}}\right\}
\end{aligned}
$$

for $\alpha \geqslant 1$, and

$$
\begin{aligned}
g(r, z ; \rho, \alpha) & =\frac{\alpha}{2 \epsilon_{0}}\left\{\frac { 2 \rho } { ( 1 - \alpha ^ { 2 } ) ^ { 1 / 2 } } \left[\tan ^{-1}\left(\frac{\eta+\alpha^{2}}{1-\alpha^{2}}\right)^{1 / 2}\right.\right. \\
& \left.-\tan ^{-1}\left(\frac{\alpha^{2}}{1-\alpha^{2}}\right)^{1 / 2}\right]+\frac{1}{\left(\eta+\alpha^{2}\right)^{1 / 2}(\eta+1) \rho} \\
& \left.\times\left(\rho^{2}-\frac{r^{2}}{\eta+1}-\frac{z^{2}}{\eta+\alpha^{2}}\right) \frac{\left(\eta+\alpha^{2}+1\right) r^{2}+(\eta+1) z^{2}}{r^{2}+z^{2}-\left(2 \eta+\alpha^{2}+1\right) \rho^{2}}\right\}
\end{aligned}
$$

for $\alpha \leqslant 1$.

If all the nested shells have the same aspect ratio and uniform charge density up to the radius $a$, the integration (10) is reduced back to Eq. (2) inside or to Eqs. (3) and (4) outside the spheroid. At the location far from the spheroid the asymptotic form of Eq. (10) leads to

$$
\begin{aligned}
\phi(r, z)= & -\frac{1}{2 \epsilon_{0}} \int_{0}^{\infty} d \rho \rho \frac{q n \alpha}{\left(\alpha^{2}-1\right)^{1 / 2}} \ln \left|\frac{\alpha+\left(\alpha^{2}-1\right)^{1 / 2}}{\alpha-\left(\alpha^{2}-1\right)^{1 / 2}}\right| \\
& +\frac{Q}{4 \pi \epsilon_{0}\left(r^{2}+z^{2}\right)^{1 / 2}}
\end{aligned}
$$

for $\alpha \geqslant 1$, and to

$$
\begin{aligned}
\phi(r, z)= & -\frac{1}{\epsilon_{0}} \int_{0}^{\infty} d \rho \rho \frac{q n \alpha}{\left(1-\alpha^{2}\right)^{1 / 2}}\left[\frac{\pi}{2}-\tan ^{-1}\left(\frac{\alpha^{2}}{1-\alpha^{2}}\right)^{1 / 2}\right] \\
& +\frac{Q}{4 \pi \epsilon_{0}\left(r^{2}+z^{2}\right)^{1 / 2}}
\end{aligned}
$$

for $\alpha \leqslant 1$. Here the charge density $q n$ and the aspect ratio $\alpha$ depend on the shell radius $\rho$, and $Q$ is the total charge. The first term stands for the height of the potential at the center of the spheroid as seen from the infinity. It should be noted that the expression of the potential $\phi^{s}(r, z ; a)$ in Eqs. (2)-(4) is taken so as $\phi^{s}(0,0 ; a)=0$ at the origin. If we choose $\phi(r, z ; a) \rightarrow 0$ at $(r, z) \rightarrow \infty$, the first term in Eq. (14) or in Eq. (15) must be subtracted from Eq. (10).

As an example of the procedure, summarized by Eqs. (10), (12), and (13), let us examine a simple model composed of two density profiles, 

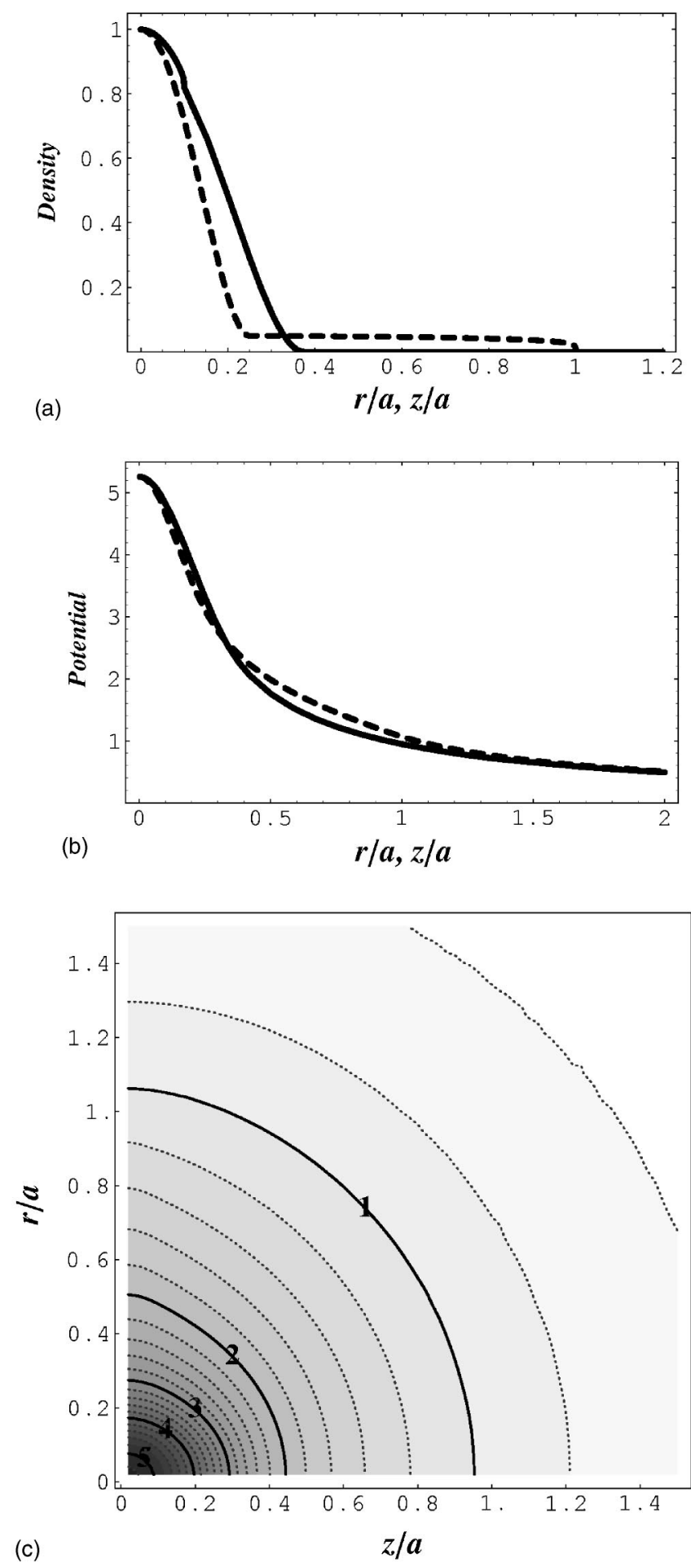

FIG. 1. (a) Normalized density profiles in the axial (solid) and radial (dashed) directions. (b) Normalized potential profiles in the axial (solid) and radial (dashed) directions. (c) Potential contours in $(r / a, z / a)$ plane.

$$
n(\rho)=n_{0}\left[f_{1} \tilde{n}_{1}(\rho)+f_{2} \tilde{n}_{2}(\rho)\right],
$$

where $n_{0}$ stands for the absolute value of the particle density, and $f_{j}$ represent the fraction of each distribution $j$. The profiles for spheroids, $j=1,2$, are assumed as

$$
\begin{aligned}
\tilde{n}_{j}(\rho) & =\left\{1-\left(\frac{r^{2}}{\rho^{2}}+\frac{z^{2}}{\alpha_{j}^{2} \rho^{2}}\right)\right\}^{\gamma_{j}}\left(\rho<a_{j}\right), \\
& =0\left(\rho>a_{j}\right) .
\end{aligned}
$$

This model consists of two distributions characterized by peaking parameter $\gamma$ and the aspect ratio $\alpha$. Various density profiles can be constructed by the combination of these parameters or by increasing the number of constituent distributions. Even a toroidal configuration may be approximated by superposing negative and positive charge distributions. Here we limit the present discussion to a distribution consisting of a slightly elongated ellipsoid 1 with $\left(a_{1}, \alpha_{1}, \gamma_{1}, f_{1}\right)$ $=(0.25 a, 1.5,2,0.95)$ and an oblate ellipsoid 2 with $\left(a_{2}, \alpha_{2}, \gamma_{2}, f_{2}\right)=(a, 0.1,0.2,0.05)$. The radial (axial) density distributions given in Eq. (16) is plotted with dashed (solid) curves in Fig. 1(a). The radial (dashed) and axial (solid) distributions of the potential are readily calculated with Eq. (10), and the results are shown in Fig. 1(b). The spatial coordinates are normalized by the radial boundary $a$. The potential is normalized by $Q / 4 \pi \epsilon_{0} a$. Figure 1(c) shows the potential contours in the $(r, z)$ plane in the region of $r<1.5 a$ and $z<1.5 a$. Figure 1 indicates that the potential distribution reflects profile structures of the density distribution in the near region of $\left(r^{2}+z^{2}\right)^{1 / 2}<2 a$.

In summary we have developed a procedure of quickly calculating the potential distribution of a 3D charge-density distribution by expressing it as the summation of sets of nested spheroids. In the application of the procedure to data analyses the fitting parameters need to be determined so that the density and the potential are consistent with observations. The analyses inevitably include iterative assignment of the parameters and repeated calculation of the potential distribution. This procedure is fast enough to perform such tasks. When applied to experimental analyses of Penning equilibria of a non-neutral plasma, the calculated potential is requested to be consistent with observations such as the axially integrated radial density distribution, potential profiles, and radially resolved energy spectra of particles. Procedures of the data analyses in actual experiments and results will be reported in separate papers.

\section{ACKNOWLEDGMENTS}

This work was supported by the Grant-in-Aid program of JSPS and partly by the collaborative research program of NIFS.

${ }^{1}$ D. H. E. Dubin and T. M. O’Neil, Rev. Mod. Phys. 71, 87 (1999).

${ }^{2}$ K. Ito, Y. Kiwamoto, and A. Sanpei, Jpn. J. Appl. Phys., Part 1 40, 2558 (2001).

${ }^{3}$ S. A. Prasad and T. M. O’Neil, Phys. Fluids 22, 278 (1979).

${ }^{4}$ E. F. Chrien, E. J. Valeo, and C. R. Oberman, Phys. Fluids 29, 1675 (1986).

${ }^{5}$ R. C. Davidson, Physics of Non-neutral Plasmas (Addison-Wesley, Redwood City, 1990), p. 116.

${ }^{6}$ L. D. Landau and E. M. Lifshits, The Classical Theory of Fields, translated by M. Hamermesh (Pergamon, New York, 1971), 3rd rev., p. 281.

${ }^{7}$ A. Mohri, H. Higaki, H. Tanaka et al., Jpn. J. Appl. Phys., Part 1 37, 664 (1998). The expression of $\beta$ after Eq. (A5) should be corrected as $\eta$ in this article. 